\title{
Deshidratación osmótica de la papaya chilena (Vasconcellea pubescens) e influencia de la temperatura y concentración de la solución sobre la cinética de transferencia de materia
}

\author{
Desidratação osmótica do mamão chileno (Vasconcellea pubescens) e influência da temperatura \\ e concentração da solução sobre a cinética de transferência de matéria
}

\author{
Antonio VEGA-GÁLVEZ ${ }^{1 *}$, Marlene PALACIOS ${ }^{1}$, Francisca BOGLIO ${ }^{1}$, \\ Catarina PÁSSARO ${ }^{2}$, Catalina JERÉZ ${ }^{1}$, Roberto LEMUS-MONDACA ${ }^{1}$
}

\begin{abstract}
Resumen
El objetivo de este trabajo fue estudiar la cinética de deshidratación osmótica de la papaya chilena (Vasconcellea pubescens), utilizando dos variables experimentales: la temperatura $\left(30,40,50{ }^{\circ} \mathrm{C}\right)$ y concentración $(40,50$ y $60 \%)$ de la solución osmótica. Se determinó la variación de masa total, masa de agua y masa de sólidos solubles y se modeló con las ecuaciones difusionales, además en esta investigación se consideró y utilizó una modificación de la ecuación propuesta por Biswal-Bozorgmehr. Estos modelos fueron evaluados por medio del coeficiente de regresión lineal, suma de errores cuadrados, raíz media de los errores cuadrados y Chi-cuadrado. Al comparar los valores experimentales con los calculados, se demostró que el modelo Biswal-Bozorgmehr modificado obtuvo mejor ajuste sobre la variación de masa de agua y sólidos solubles. Mediante la ecuación de Arrhenius se analizó el efecto de la temperatura sobre los parámetros cinéticos $\left(\mathrm{K}_{\mathrm{w}}\right.$ y $\left.\mathrm{K}_{\mathrm{ss}}\right)$ y difusividad efectiva de agua $\left(\mathrm{D}_{\text {we }}\right)$ y sólidos solubles $\left(\mathrm{D}_{\mathrm{sse}}\right)$. De acuerdo al diseño factorial, se observó que la temperatura no influyó sobre la difusividad efectiva de agua y de sólidos, pero sí la concentración de la solución osmótica. La mejor condición de salida de agua y ganancia de sólidos solubles durante la deshidratación osmótica de la papaya chilena fue a $30{ }^{\circ} \mathrm{C}$ con una concentración del $60 \%$.

Palabras-Clave: papaya chilena; difusividad efectiva; modelos cinéticos.
\end{abstract}

\section{Resumo}

O objetivo deste trabalho foi estudar a cinética de desidratação osmótica do mamão chileno (Vasconcellea pubescens) utilizando duas variáveis experimentais: temperatura $\left(30,40\right.$ e $\left.50^{\circ} \mathrm{C}\right)$ e concentração $(40,50$ e $60 \%)$ da solução osmótica. Para este estudo, determinou-se a variação de massa total, de massa de água, de massa de sólidos solúveis, modeladas com as equações difusionais. Nesta investigação, também se considera e se usa uma modificação da equação proposta por Biswal-Bozorgmehr. Estes modelos foram avaliados através do coeficiente de regressão linear, da somatória de erros quadrados, da raiz média de erros quadrados e do Qui-quadrado. Comparando os valores experimentais com os valores calculados, demonstrou-se que o modelo Biswal-Bozorgmehr modificado apresenta um melhor ajuste sobre a variação da massa de água e de sólidos solúveis. Com a equação de Arrhenius, analisou-se o efeito da temperatura sobre os parâmetros cinéticos ( $\mathrm{K}_{\mathrm{w}}$ e $\left.\mathrm{K}_{\mathrm{ss}}\right)$ e a difusividade efetiva de água $\left(\mathrm{D}_{\mathrm{we}}\right)$ e de sólidos solúveis $\left(\mathrm{D}_{\mathrm{sse}}\right)$. De acordo com o desenho fatorial, observou-se que a temperatura não teve influência sobre a difusividade efetiva da água e dos sólidos solúveis, mas sim sobre a concentração da solução osmótica. A melhor condição de saída de água e ganho de sólidos durante a desidratação osmótica do mamão chileno foi a $30{ }^{\circ} \mathrm{C}$ com uma concentração de $60 \%$.

Palavras-chave: mamão chileno; difusividade efetiva; modelos cinéticos.

\section{Introducción}

A diferencia de la papaya tropical (Carica papaya L.) cultivada en Brasil (var. Formosa), Colombia (var. Maradol) y países del Caribe (var. Hawaiana) $)^{6,8,12}$, la papaya chilena (Vasconcellea pubescens) es única a nivel mundial. Esta se cultiva y crece en climas más fríos y con producción máxima entre Noviembre y Febrero. Es más pequeña y firme, con pulpa de color verde amarillo, su atractivo sensorial es su fuerte y característico aroma. Tiene un rendimiento comestible del $46 \%$ y un contenido de azúcar de $5 \%$, además se caracteriza principalmente por su alto contenido en papaína ${ }^{18}$.

La deshidratación osmótica (DO) consiste en la inmersión de un alimento sólido, entero o en piezas, en soluciones acuosas de alta concentración en solutos (hipertónica) a un tiempo

Recebido para publicação em 30/3/2006

Aceito para publicação em 18/7/2007 (001720)

Departamento de Ingeniería en Alimentos, Universidad de La Serena, Box 599

La Serena, Chile,

E-mail: avegag@userena.cl

Instituto Valenciano de Investigaciones Agrarias, Carretera Moncada-Náquera,

$s / n$, Box 46113, Valencia, España

*A quem a correspondência deve ser enviada y temperaturas específicos. Las membranas de los alimentos son semipermeables por lo cual esta técnica provoca al menos dos flujos principales simultáneos en contracorriente. Un importante flujo de agua del interior de la fruta hacia el exterior, para tratar de equilibrar el potencial químico del agua a ambos lados de dichas membranas. Simultáneamente se presenta, en menor cantidad, la entrada de soluto desde la parte externa hacia el interior del producto a deshidratar ${ }^{19}$. Además hay otro flujo que se debe considerar el que consiste en una mínima pérdida de solutos propios del alimento (azúcares, sales minerales, ácidos orgánicos y otros) que aunque cuantitativamente es insignificante, puede tener alguna importancia a nivel nutricional y organoléptico ${ }^{7}$.

La DO es un método que, reduciendo hasta un $80 \%$ del agua original de los alimentos, permite obtener productos de humedad intermedia, con una buena calidad organoléptica. La solución osmótica que se usa para deshidratar el producto debe ser rica en solutos que depriman la actividad de agua del mismo, y que genere una diferencia de presión osmótica entre el producto a deshidratar y la solución ${ }^{5}$. Los solutos que normalmente se utilizan en las soluciones osmóticas, son de 
bajo costo, tales como sacarosa, glucosa, fructosa, cloruro de sodio, glicerol, sorbitol y combinaciones de estos, presentando efecto sinérgico, como es el caso de la mezcla sacarosa-cloruro de sodio ${ }^{11}$. Generalmente, las soluciones de sacarosa son usadas para frutas y las soluciones de cloruro de sodio para vegetales $^{1}$.

Existen estudios de las principales variables que producen efecto sobre la deshidratación osmótica, específicamente sobre la cinética de transferencia de materia, entre ellas destacan las propias del producto como composición, tamaño, forma, presencia de piel, pretratamientos previos $^{5}$, y de la solución osmótica como temperatura, concentración, naturaleza del agente osmótico, presión de trabajo, razón alimento-solución, tiempo y agitación ${ }^{9,11}$.

El objetivo de este trabajo fue estudiar y modelar la cinética de proceso de la deshidratación osmótica de la papaya chilena y evaluar la influencia de la concentración y temperatura de la solución sobre la cinética de transferencia de materia (agua y solutos).

\section{Materiales y métodos}

\subsection{Materia prima}

Los frutos de papaya (Vasconcellea pubescens) se adquirieron en el mercado local de la ciudad La Serena (Chile), seleccionados utilizando siempre el mismo criterio, es decir, similar estado de madurez, tamaño, firmeza y color. Los frutos se conservaron a $7,0 \pm 1,0^{\circ} \mathrm{C}$ hasta su procesamiento. Luego se pelaron utilizando una solución de soda cáustica $(\mathrm{NaOH} 10 \%$ y FastPeel ${ }^{\circledR} 1 \%$ ) a una temperatura de $70{ }^{\circ} \mathrm{C}$, inmediatamente se lavaron con agua fría para retirar los restos de piel, sin producir daños en los tejidos. El despepitado y cortado se realizó en forma manual obteniendo cubitos de 1,0 $\pm 0,1 \mathrm{~cm}$ de lado.

\subsection{Diseño experimental}

El diseño experimental empleado esta basado en un diseño factorial $\mathrm{n}^{\mathrm{k}}$, donde $\mathrm{n}$ es el numero de niveles y $\mathrm{k}$ es el numero de factores, donde temperatura y concentración son los dos factores a estudiar $(\mathrm{k}=2)$, cada uno con tres niveles $(\mathrm{n}=3)$, por lo tanto, se requirieron de 9 experimentos $\left(3^{2}\right)$. La Tabla 1 muestra los niveles decodificados de las variables a utilizar en el diseño experimental para luego representar los experimentos en la Tabla 2 ya codificados ${ }^{2}$.

Tabla 1. Niveles decodificados de las variables.

\begin{tabular}{ccc}
\hline \multicolumn{3}{c}{ Niveles decodificados } \\
\hline Niveles codificados & $\begin{array}{c}\text { Concentración de la } \\
\text { solución }(\%)\end{array}$ & $\begin{array}{c}\text { Temperatura de la } \\
\text { solución }\left({ }^{\circ} \mathrm{C}\right)\end{array}$ \\
\hline-1 & 40 & 30 \\
0 & 50 & 40 \\
+1 & 60 & 50 \\
\hline
\end{tabular}

\subsection{Deshidratación osmótica}

Se prepararon soluciones osmóticas de sacarosa comercial (marca IANSA) a tres concentraciones distintas (40,50 y
Tabla 2 - Matriz de diseño experimental codificada $\left(3^{2}\right)$.

\begin{tabular}{ccc}
\hline Experimento & $\mathrm{C}(\%)$ & $\mathrm{T}\left({ }^{\circ} \mathrm{C}\right)$ \\
\hline 1 & -1 & -1 \\
2 & 0 & -1 \\
3 & +1 & -1 \\
4 & -1 & 0 \\
5 & 0 & 0 \\
6 & +1 & 0 \\
7 & -1 & +1 \\
8 & 0 & +1 \\
9 & +1 & +1 \\
\hline
\end{tabular}

$60 \%)$, las que fueron controladas usando un refractómetro (marca ATAGO). Las temperaturas de trabajo fueron 30, 40 y $50{ }^{\circ} \mathrm{C}$. Los trozos de papaya fueron sumergidos en distintos vasos precipitados de $2000 \mathrm{~mL}$ cada uno, los que contenían la solución osmótica. Se empleó una proporción fruta/solución de 1:5. Los recipientes se introdujeron en un baño de agua termorregulado (marca MEMMERT) para controlar y mantener constante la temperatura de trabajo, la que fue monitoreada usando un termómetro digital (marca HANNA INSTRUMENT) de precisión $\pm 0,1^{\circ} \mathrm{C}$. Para el estudio de la variación de masa, las muestras identificadas fueron pesadas cada cierto intervalo de tiempo: 0, 5, 10, 15, 30 minutos, a partir de este último tiempo las muestras fueron retiradas de la solución osmótica cada 30 minutos hasta las 7 horas. Todos los experimentos se hicieron en triplicado.

\subsection{Determinaciones analíticas}

El contenido de humedad de las muestras a cada intervalo de tiempo se determinó por el método gravimétrico oficial de A.O.A.C. ${ }^{3}$. Posteriormente, fueron depositadas en placas Petri, las que fueron colocadas en una estufa a vacío (marca GALLENKAMP) a $60{ }^{\circ} \mathrm{C}$ a una presión de $10 \mathrm{~mm} \mathrm{Hg}$ por 72 horas hasta alcanzar peso constante. Una balanza analítica (marca CHYO Jex120) de precisión 0,01 g se utilizó. Los análisis fueron realizados en triplicado informando los valores medios y su desviación estándar. La determinación del contenido de sólidos solubles presentes en las muestras, se realizó mediante un refractómetro de mesón (marca ATAGO), en el cual se colocó una gota de líquido extraído de la fruta, haciendo pasar la luz a través de él, observándose, en una escala graduada. Los resultados se expresaron como ${ }^{\circ} \mathrm{Brix}$ a $20^{\circ} \mathrm{C}$.

\subsection{Ecuaciones utilizadas en el estudio de la transferencia de materia}

La variación de masa total $(\Delta \mathrm{M})$, pérdida de agua $\left(\Delta \mathrm{M}_{\mathrm{w}}\right)$ y ganancia de sólidos solubles $\left(\Delta \mathrm{M}_{\mathrm{ss}}\right)$, se calculó usando la Ecuaciones 1, 2 y 3, respectivamente ${ }^{6,16}$.

$$
\begin{aligned}
& \Delta \mathrm{M}=\frac{\mathrm{M}_{\mathrm{t}}-\mathrm{M}_{0}}{\mathrm{M}_{0}} \\
& \Delta \mathrm{M}_{\mathrm{w}}=\frac{\mathrm{M}_{\mathrm{t}} \mathrm{X}_{\mathrm{wt}}-\mathrm{M}_{0} \mathrm{X}_{\mathrm{w} 0}}{\mathrm{M}_{0}}
\end{aligned}
$$


$\Delta \mathrm{M}_{\mathrm{ss}}=\frac{\mathrm{M}_{\mathrm{t}} \mathrm{X}_{\mathrm{sst}}-\mathrm{M}_{0} \mathrm{X}_{\mathrm{ss} 0}}{\mathrm{M}_{0}}$

Para el modelado de la pérdida de humedad y la ganancia de sólidos solubles del tiempo de inmersión en la solución osmótica, se aplicó el modelo propuesto por Biswal-Bozorgmehr $(1992)^{13}$. Con este trabajo se plantea que dicho modelo puede ser modificado de acuerdo a las siguientes Ecuaciones 4 y 5 , al cual se le llamará modelo Biswal-Bozorgmehr modificado.

$\Delta \mathrm{M}_{\mathrm{w}}=\mathrm{K}_{\mathrm{w}} \mathrm{t}^{0,5}$

$\Delta \mathrm{M}_{\mathrm{ss}}=\mathrm{K}_{\mathrm{ss}} \mathrm{t}^{0,5}$

Los valores de difusividad efectiva $\left(\mathrm{D}_{\mathrm{e}}\right)$ de agua y de sólidos, fueron obtenidos aplicando las ecuaciones difusionales integrando la segunda ley de Fick, para tiempos largos de proceso y suponiendo los cubitos de papaya como una lámina infinita. Debido a que los datos experimentales cumplen las condiciones de entorno (geometría del producto, concentración inicial homogénea, concentración constante de la interfase) la ecuación de Fick (6) converge rápidamente pudiéndose utilizar solo el primer término del desarrollo de la serie como una buena aproximación $(7)^{8,12}$. Para el cálculo de la difusividad $\left(D_{\mathrm{e}}\right)$ se linealiza dicha ecuación y se convierte en la Ecuación 8, donde la pendiente de la ecuación obtenida se iguala a $-\mathrm{D}_{\mathrm{e}} \pi^{2} / 4 \mathrm{~L}^{2}$, de donde se obtiene la $\mathrm{D}_{\mathrm{e}}$. Para determinar la dependencia de la difusividad efectiva con la temperatura, se utilizó la ecuación de Arrhenius (9) ${ }^{17}$ y la energía de activación fue obtenida linealizando la misma Ecuación (10).

$\mathrm{Y}=\frac{\mathrm{X}_{\mathrm{wt}}-\mathrm{X}_{\mathrm{we}}}{\mathrm{X}_{\mathrm{wo}}-\mathrm{X}_{\mathrm{we}}}=1-\sum_{\mathrm{n}=0}^{\infty} \frac{8}{(2 \mathrm{n}+1)^{2} \pi^{2}} \exp \left\{-\mathrm{D}_{\mathrm{e}}(2 \mathrm{n}+1)^{2} \frac{\pi^{2} \mathrm{t}}{4 \mathrm{~L}^{2}}\right\}$

$\mathrm{Y}=\frac{8}{\pi^{2}} \exp \left(\frac{-\mathrm{D}_{\mathrm{e}} \pi^{2} \mathrm{t}}{4 \mathrm{~L}^{2}}\right)$

$\operatorname{Ln} Y=\operatorname{Ln} \frac{8}{\pi^{2}}-\left(\frac{D_{\mathrm{e}} \pi^{2}}{4 \mathrm{~L}^{2}}\right) \cdot \mathrm{t}$

$\mathrm{D}_{\mathrm{e}}=\mathrm{D}_{\mathrm{o}} \cdot \exp \left(-\mathrm{E}_{\mathrm{a}} / \mathrm{R} \cdot \mathrm{T}\right)$

$\operatorname{LnD}_{\mathrm{e}}=\mathrm{LnD}_{\mathrm{o}}-\frac{\mathrm{E}_{\mathrm{a}}}{\mathrm{RT}}$

\subsection{Análisis estadístico}

Para el análisis estadístico de los datos experimentales se utilizó el software Statgraphics Plus ${ }^{\circledR}$ para Windows versión 5.1. La significación estadística de los efectos fue analizada mediante un análisis de varianza (ANOVA) simple y factorial ( $p<0,05$ ), además se realizó una regresión múltiple para ver la interrelación entre las variables estudiadas. Todos estos análisis fueron realizados con un nivel de confianza de 95,0\%. Para evaluar la calidad de ajuste obtenido de los modelos propuestos sobre los datos experimentales, se utilizaron: el coeficiente de regresión lineal $\left(\mathrm{r}^{2}\right)$, suma de los errores cuadrados (SSE) (11), la raíz media de los errores cuadrados (RMSE) (12) y el parámetro Chi-cuadrado $\left(\chi^{2}\right)$ (13). Los valores más bajos de SEE, RMSE y $\chi^{2}$ o que tiendan a cero, junto con los más altos valores de $\mathrm{r}^{2}$ o que tiendan a uno, se consideran como óptimos y ayudan a elegir el mejor modelo ${ }^{18}$.

$\mathrm{SSE}=\frac{1}{\mathrm{~N}} \sum_{\mathrm{i}=1}^{\mathrm{N}}\left(\mathrm{Y}_{\mathrm{e}}-\mathrm{Y}_{\mathrm{c}}\right)^{2}$

$\mathrm{RMSE}=\left[\frac{1}{\mathrm{~N}} \sum_{\mathrm{i}=1}^{\mathrm{N}}\left(\mathrm{Y}_{\mathrm{c}}-\mathrm{Y}_{\mathrm{e}}\right)^{2}\right]^{1 / 2}$

$\chi^{2}=\frac{\sum_{\mathrm{i}=1}^{\mathrm{N}}\left(\mathrm{Y}_{\mathrm{e}}-\mathrm{Y}_{\mathrm{c}}\right)^{2}}{\mathrm{~N}-\mathrm{z}}$

\section{Resultados y discusión}

\subsection{Comprobación de balance de masa}

La ecuación 14 define la relación existente entre la concentración de los componentes a un tiempo determinado, asumiendo que la variación de masa es debida únicamente al flujo de agua desde el producto al exterior (solución osmótica) y a la ganancia de solutos por parte de la fruta desde la solución.

$\Delta \mathrm{M}=\Delta \mathrm{M}_{\mathrm{w}}+\Delta \mathrm{M}_{\mathrm{ss}}$

La Figura 1 muestra que el supuesto anteriormente mencionado es correcto, validado esto con la comprobación del balance de masa total realizado para cada temperatura y concentración estudiada. Para todas las series de temperatura y concentración, prácticamente se observa un comportamiento lineal con un alto valor del coeficiente de correlación $\left(r^{2} \geq 0,90\right)$. Estos resultados coinciden con lo propuesto por BARAT et al. ${ }^{4}$.

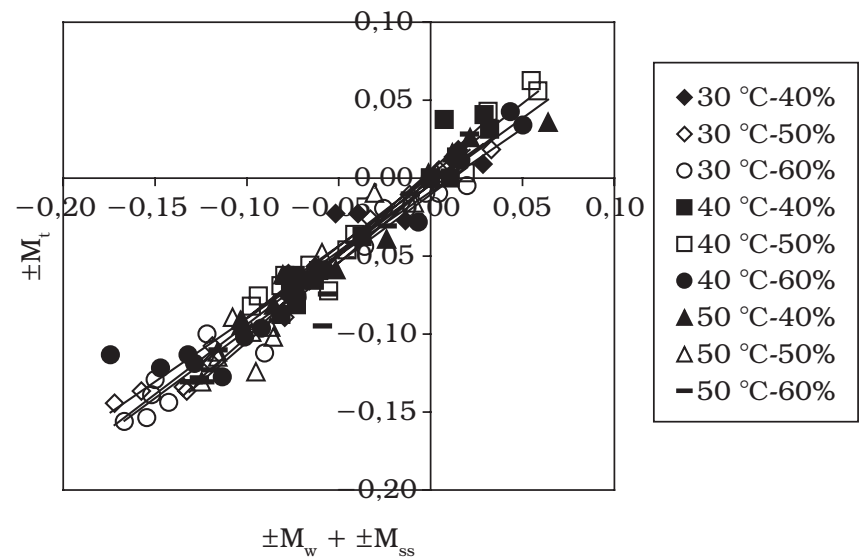

Figura 1. Comprobación de balance de masa total en tiempo total de inmersión (7 horas), para las diferentes temperaturas y concentraciones utilizadas.

\subsection{Variación de masa de agua y de sólidos en función del tiempo}

La Figura 2 muestra que la pérdida de agua y la ganancia de sólidos aumentan a medida que aumenta el tiempo de tratamiento, similares resultados obtuvo SABLANI, SHAFIUR y AL-SADEIRI ${ }^{15}$ estudiando la deshidratación osmótica de 
manzana. En la Figura 2, además se aprecia que a medida que aumenta la concentración de la solución, mayor es la variación de masa de agua, siendo la más alta a una concentración de $60 \%$, para todas las temperaturas de trabajo.

En relación a los sólidos solubles en los primeros minutos se observó un estado de pseudoequilibrio donde hay una tendencia a ganar sólidos solubles para las distintas concentraciones utilizadas, haciéndose esto notorio para una concentración de $60 \%$, probablemente debido a la rápida acción de los mecanismos hidrodinámicos que provoca la ganancia inicial de la solución osmótica por capilaridad o por los cambios de presión impuestos ${ }^{10}$, sin embargo a partir de los 15 minutos todas las curvas tienden a ganar sólidos gradualmente.

En general, para todas las temperaturas de trabajo se observa la marcada influencia de la concentración sobre la ganancia de sólidos solubles, debido a que mayor concentración de sacarosa, mayor es la ganancia de sólidos solubles, lo mismo propone GASPARETO, OLIVEIRA y MAGALHAES ${ }^{9}$ en la deshidratación osmótica de la banana; y RASTOGI y RAGHAVARAO ${ }^{14}$ con el estudio de deshidratación osmótica en piña.

\subsection{Cálculo de los parámetros cinéticos $K_{w}$ y $K_{s s}$}

De acuerdo a los valores obtenidos en la Tabla 3, se observa que la concentración de la solución osmótica es más influyente que la temperatura en la cinética de pérdida de agua $\left(\mathrm{K}_{\mathrm{w}}\right) \mathrm{y}$ de ganancia de sólidos solubles $\left(\mathrm{K}_{\mathrm{ss}}\right)$. Ya que con el aumento de la concentración para todas las temperatura de trabajo, el valor absoluto promedio de estas constantes $\left(\mathrm{K}_{\mathrm{w}} \mathrm{y} \mathrm{K}_{\mathrm{ss}}\right)$ va en aumento.

Tabla 3. Valores de los parámetros cinéticos $\mathrm{K}_{\mathrm{w}} \mathrm{y} \mathrm{K}_{\mathrm{ss}}$ para cada experimento.

\begin{tabular}{ccc}
\hline Experimento & $\mathrm{K}_{\mathrm{w}} \cdot 10^{-3}$ & $\mathrm{~K}_{\mathrm{ss}} \cdot 10^{-3}$ \\
\hline 1 & $-13,78 \pm 0,12$ & $10,21 \pm 0,18$ \\
2 & $-17,78 \pm 0,17$ & $13,77 \pm 0,18$ \\
3 & $-18,31 \pm 0,14$ & $13,54 \pm 0,13$ \\
4 & $-20,50 \pm 0,10$ & $12,40 \pm 0,05$ \\
5 & $-20,14 \pm 0,22$ & $16,02 \pm 0,01$ \\
6 & $-23,83 \pm 0,29$ & $17,19 \pm 0,08$ \\
7 & $-23,65 \pm 0,11$ & $15,11 \pm 0,20$ \\
8 & $-26,60 \pm 0,19$ & $18,99 \pm 0,21$ \\
9 & $-27,20 \pm 0,08$ & $19,01 \pm 0,22$ \\
\hline
\end{tabular}

Al evaluar la influencia de la temperatura sobre las constantes cinéticas $\mathrm{K}_{\mathrm{w}} \mathrm{y} \mathrm{K}_{\mathrm{ss}}$, mediante la ecuación Arrhenius (Figura 3a y 3b), se pudo observar una cierta dependencia de la temperatura sobre $\mathrm{K}_{\mathrm{w}}$ para una concentración de 40 y $60 \%$ dado que su valor $r^{2} \geq 0,84$, no así para una concentración del $50 \%$, donde el coeficiente de correlación fue muy bajo (Figura 3a). Sin embargo, al analizar estadísticamente el factor temperatura mediante el test de las menores diferencias significativas de Fisher (Least Significant Difference, LSD), éste arrojo un valor $p>0,05$, no existiendo diferencia estadísticamente significativa entre las medias de $\mathrm{K}_{\mathrm{w}}$ para todas las concentraciones de trabajo. (a)

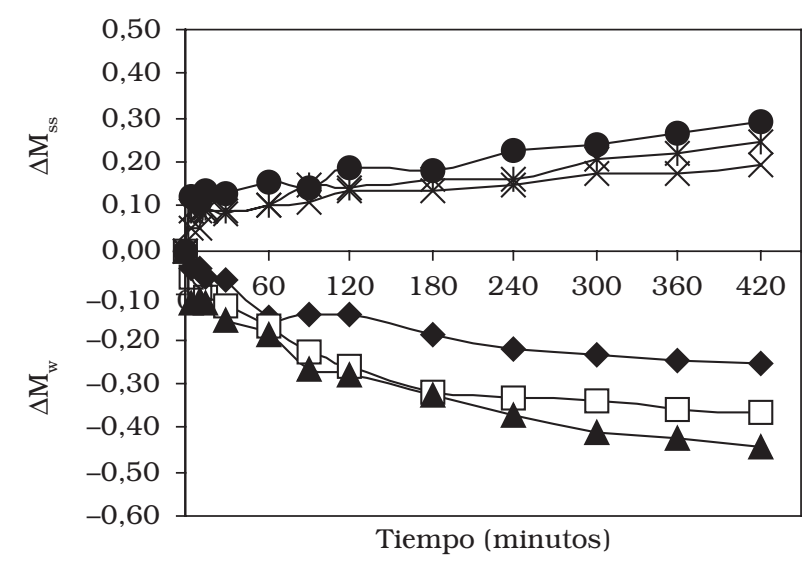

$\neg-40 \%$ agua $\square-50 \%$ agua $\longrightarrow 60 \%$ agua $\longrightarrow \quad 40 \%$ sacarosa $\longrightarrow-50 \%$ sacarosa $\longrightarrow-60 \%$ sacarosa

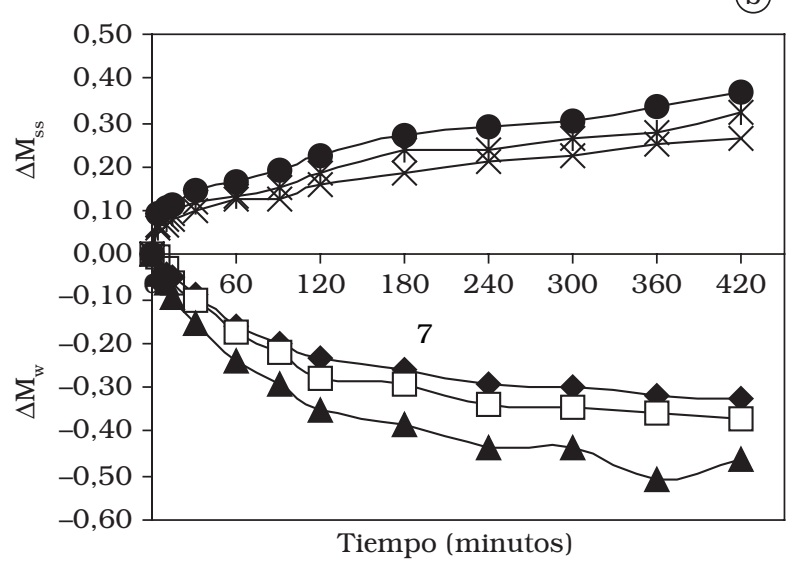

$\begin{array}{lll}\longrightarrow-40 \% \text { agua } & -\square-50 \% \text { agua } & -60 \% \text { agua } \\ \times-40 \% \text { sacarosa } & -650 \% \text { sacarosa } & -60 \% \text { sacarosa }\end{array}$

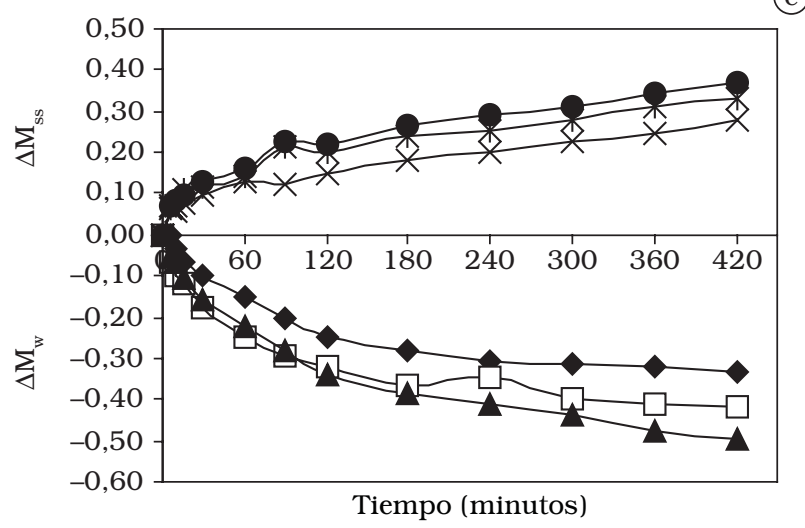

(c)

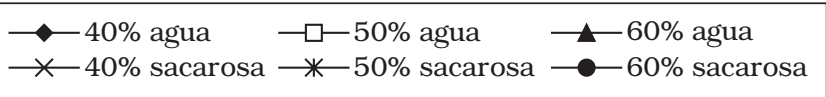

Figura 2. a) Cambios en el contenido de agua $\left(\Delta \mathrm{M}_{\mathrm{w}}\right)$ y sólidos solubles $\left(\Delta \mathrm{M}_{\mathrm{ss}}\right)$, durante el tratamiento osmótico a $30^{\circ} \mathrm{C}$; b) $40{ }^{\circ} \mathrm{C}$; y c) $50{ }^{\circ} \mathrm{C}$ en tiempo total de inmersión. 

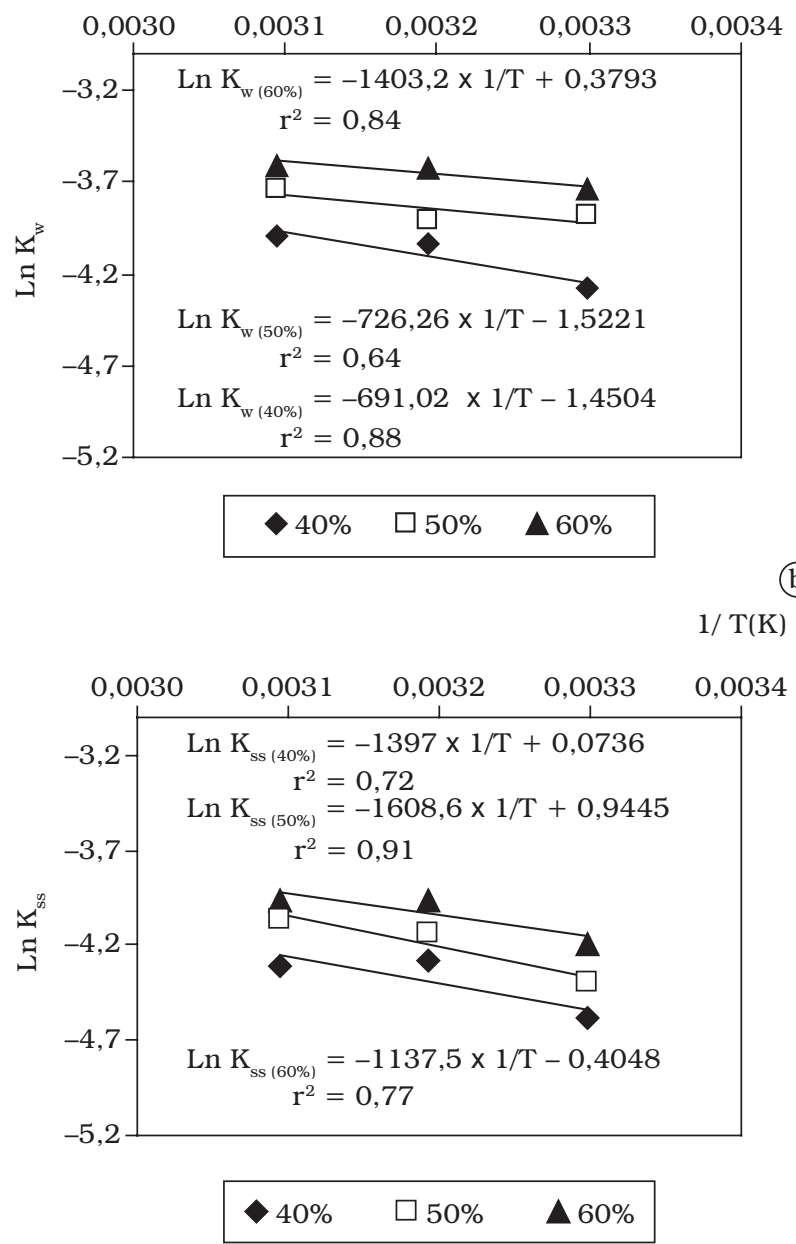

Figura 3. a) Gráfico de Arrhenius para las constantes cinéticas $K_{w}$; y b) $\mathrm{K}_{\mathrm{ss}}$.

En cambio al analizar con la misma técnica estadística (LSD) la influencia del factor concentración, sobre la constante $\mathrm{K}_{\mathrm{w}}$, se encontró un valor $\mathrm{p}<0,05$, por lo tanto existe diferencia estadísticamente significativa entre las medias de $\mathrm{K}_{\mathrm{w}}$. Esto se puede corroborar al observar la misma gráfica (Figura 3a) debido a que las curvas no se entrecruzan.

Para el caso de los sólidos, la Figura 3b muestra que prácticamente no existe dependencia de la temperatura sobre la constante cinética $\mathrm{K}_{\mathrm{ss}}$ para una concentración de 40 y $60 \%$, ya que sus valores de $\mathrm{r}^{2}$ son inferiores a 0,80 . Sin embargo, para una concentración de $50 \%$ el coeficiente de correlación $\mathrm{r}^{2}$ es superior a 0,90, lo cual indicaría que existe una cierta dependencia con esta variable física. Este análisis se corroboró mediante un análisis LSD, el cual arrojo un valor $\mathrm{p}>0,05$ para el factor temperatura, no habiendo diferencia estadísticamente significativa entre las medias de $\mathrm{K}_{\mathrm{ss}}$. Por otro lado, al analizar la influencia de la concentración sobre $\mathrm{K}_{\mathrm{ss}}$ utilizando la misma técnica (LSD), se obtuvo un valor $\mathrm{p}<0,05$, lo que indica que existe diferencia estadísticamente significativa entre las medias de $\mathrm{K}_{\mathrm{ss}}$.
Si bien es cierto, al evaluar la influencia de la temperatura sobre las constantes cinéticas $\mathrm{K}_{\mathrm{w}} \mathrm{y} \mathrm{K}_{\mathrm{ss}}$, mediante la ecuación Arrhenius (Figura 3a y 3b), éstas no presentaron una marcada dependencia con ésta variable física, igual se calculó la energía de activación de las mismas. La Tabla 4 muestra los valores de la energía de activación para las constantes $\mathrm{K}_{\mathrm{w}} \mathrm{y} \mathrm{K}_{\mathrm{ss}}$, además, se puede observar que a medida que aumenta la concentración disminuye la $\mathrm{E}_{\mathrm{a}}$, para el $\mathrm{K}_{\mathrm{w}}$, nuevamente confirmando que este factor influye sobre dicho parámetro.

Tabla 4. Valores de energía de activación y constante difusional para las constantes cinéticas $\mathrm{K}_{\mathrm{w}} \mathrm{y} \mathrm{K}_{\mathrm{ss}}$ para las diferentes concentraciones de trabajo.

\begin{tabular}{cccccc}
\hline $\mathrm{C}(\%)$ & $\mathrm{K}_{\mathrm{w}}$ & & & $\mathrm{K}_{\mathrm{ss}}$ \\
\cline { 2 - 3 } \cline { 5 - 6 } & $\mathrm{E}_{\mathrm{a}}\left(\mathrm{kJ} \cdot \mathrm{mol}^{-1}\right)$ & $\mathrm{K}_{\mathrm{wo}}\left(\mathrm{m}^{2} \cdot \mathrm{s}^{-1}\right)$ & & $\mathrm{E}_{\mathrm{a}}\left(\mathrm{kJ} \cdot \mathrm{mol}^{-1}\right)$ & $\mathrm{K}_{\mathrm{sso}}\left(\mathrm{m}^{2} \cdot \mathrm{s}^{-1}\right)$ \\
\hline 40 & 11,67 & 1,46 & & 11,62 & 1,08 \\
50 & 6,04 & 0,22 & & 13,37 & 2,573 \\
60 & 5,75 & 0,24 & & 9,46 & 0,67 \\
\hline
\end{tabular}

\subsection{Cálculo de los parámetros cinéticos $D_{w e}$ y $D_{s s e}$}

La Tabla 5 muestra los valores de difusividad efectiva agua $\left(\mathrm{D}_{\mathrm{we}}\right)$ y de sólidos solubles $\left(\mathrm{D}_{\mathrm{sse}}\right)$ obtenidos para las diferentes temperaturas y concentraciones de trabajo. Ambas difusividades, presentan una clara dependencia positiva con la concentración de la solución osmótica, esto es, los valores de $\mathrm{D}_{\text {we }} \mathrm{y}_{\text {sse }}$ aumentan significativamente a medida que aumenta la concentración. Sin embargo, la influencia de la temperatura sobre estos parámetros no es del todo clara. Al comparar los valores de difusividad efectiva de agua $\left(\mathrm{D}_{\text {we }}\right)$ con los obtenidos en el estudio de deshidratación osmótica de piña RASTOGI y RAGHAVARAO ${ }^{14}$ para las mismas concentraciones y temperaturas de trabajo, se obtuvieron valores muy similares.

Tabla 5. Valores medios y desviación estándar de $\mathrm{D}_{\text {we }}$ y $\mathrm{D}_{\text {sse }}$ para cada experimento.

\begin{tabular}{crr}
\hline Experimento & $\mathrm{D}_{\mathrm{we}} \cdot 10^{-10}\left(\mathrm{~m}^{2} \cdot \mathrm{s}^{-1}\right)$ & \multicolumn{1}{c}{$\mathrm{D}_{\mathrm{sse}} \cdot 10^{-10}\left(\mathrm{~m}^{2} \cdot \mathrm{s}^{-1}\right)$} \\
\hline 1 & $9,53 \pm 0,63$ & $6,55 \pm 0,66$ \\
2 & $18,19 \pm 4,44$ & $9,88 \pm 1,93$ \\
3 & $40,43 \pm 2,22$ & $15,80 \pm 2,08$ \\
4 & $12,07 \pm 0,22$ & $6,42 \pm 0,20$ \\
5 & $19,56 \pm 2,15$ & $9,81 \pm 1,92$ \\
6 & $32,87 \pm 5,40$ & $18,58 \pm 2,68$ \\
7 & $11,85 \pm 0,33$ & $5,54 \pm 0,26$ \\
8 & $21,37 \pm 0,25$ & $10,70 \pm 0,39$ \\
9 & $31,28 \pm 2,31$ & $19,32 \pm 1,70$ \\
\hline
\end{tabular}

Con respecto a los valores de difusividad efectiva de sólidos solubles $\left(\mathrm{D}_{\mathrm{sse}}\right)$ podemos observar que la concentración de la solución también es más influyente que la temperatura de trabajo, ya que al aumentar la concentración de sacarosa mayor es la difusividad de sólidos solubles, lo mismo se obtuvo para deshidratación osmótica de piña donde la difusividad de sólidos solubles incrementa con un aumento en la concentración de la solución osmótica debido a los cambios en las propiedades físicas de los alimentos, tales como, porosidad y permeabilización de las células ${ }^{14}$. 
$\mathrm{Al}$ analizar la influencia de la temperatura sobre la difusividad efectiva de agua $\left(\mathrm{D}_{\text {we }}\right)$ y de sólidos solubles $\left(\mathrm{D}_{\mathrm{sse}}\right)$ mediante la ecuación Arrhenius (Figura 4a y 4b), se observa en general que no existe dependencia con la temperatura, lo que se corroboró con el análisis estadístico de los datos, donde el factor temperatura obtuvo un valor $\mathrm{p}>0,05$, para ambas difusividades, no existiendo diferencia estadísticamente significativa entre las medias de $\mathrm{D}_{\text {we }} \mathrm{y}_{\text {sse }}$. Sin embargo, para el factor concentración de la solución osmótica se obtuvo un valor $\mathrm{p}<0,05$ indicando diferencia estadísticamente significativa entre las medias de $\mathrm{D}_{\text {we }}$ $\mathrm{y} \mathrm{D}_{\text {sse }}$ para todas las temperaturas de trabajo.
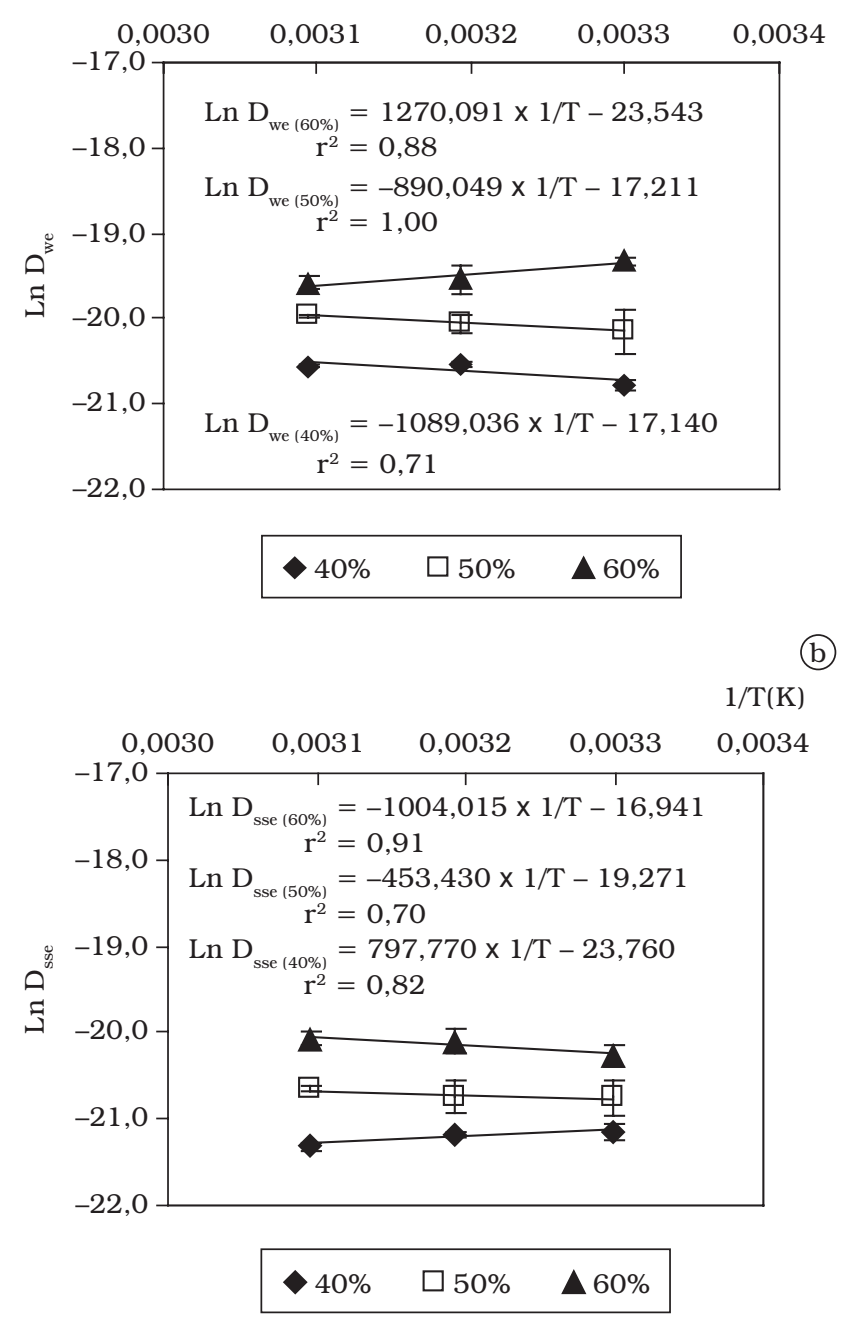

Figura 4. a) Gráfico de Arrhenius para difusividad efectiva de agua y b) sólidos solubles.

Los valores de $\mathrm{E}_{\mathrm{a}}$ para la difusividad de agua y sólidos se muestran en la Tabla 6. Resultados similares fueron obtenidos por otros autores, como MENDOZA y SCHMALKO ${ }^{12}$ en su estudio con papaya var. Hawaiana, con valores de $\mathrm{D}_{\text {we }}$ $\mathrm{y} \mathrm{D}_{\text {sse }}$ de $1,30 \times 10^{-9}$ y $3,47 \times 10^{-9} \mathrm{~m}^{2} \cdot \mathrm{s}^{-1}$, respectivamente ${ }^{12}$. No obstante, se puede observar que la concentración al $50 \%$ mostró la menor energía de activación, en ambos parámetros cinéticos $\left(\mathrm{D}_{\mathrm{we}} \mathrm{y} \mathrm{D}_{\text {sse }}\right)$, corroborando la influencia mucho mayor de la concentración que de la temperatura sobre la cinética de transferencia de materia de agua y sólidos en la deshidratación osmótica de la papaya.

Tabla 6. Valores de energía de activación y constante difusional para $\mathrm{D}_{\mathrm{we}}$ y $\mathrm{D}_{\mathrm{sse}}$ para las diferentes concentraciones de trabajo.

\begin{tabular}{cccccc}
\hline $\mathrm{C}$ & \multicolumn{2}{c}{$\mathrm{D}_{\mathrm{we}}$} & & \multicolumn{2}{c}{$\mathrm{D}_{\mathrm{sse}}$} \\
\cline { 5 - 6 } \cline { 5 - 6 } \cline { 5 - 6 } & $\mathrm{E}_{\mathrm{a}}\left(\mathrm{kJ} \cdot \mathrm{mol}^{-1}\right)$ & $\mathrm{D}_{\mathrm{wo}} \times 10^{-8}\left(\mathrm{~m}^{2} \cdot \mathrm{s}^{-1}\right)$ & & $\mathrm{E}_{\mathrm{a}}\left(\mathrm{kJ} \cdot \mathrm{mol}^{-1}\right)$ & $\mathrm{D}_{\mathrm{sso}} \times 10^{-9}\left(\mathrm{~m}^{2} \cdot \mathrm{s}^{-1}\right)$ \\
\hline 40 & 10,56 & 0,60 & & 6,63 & 0,05 \\
50 & 7,40 & 3,35 & & 3,77 & 4,27 \\
60 & 9,05 & 3,60 & & 8,35 & 43,90 \\
\hline
\end{tabular}

\subsection{Análisis estadístico de los modelos propuestos}

$\mathrm{Al}$ modelar la variación de masa de agua $\left(\Delta \mathrm{M}_{\mathrm{w}}\right)$ con el modelo de Biswal-Bozorgmehr modificado y analizar los resultados de las pruebas estadísticas aplicadas, se obtiene un $r^{2} \geq 0,85$ para todos los experimentos realizados, sin embargo, se obtuvo un valor de $\mathrm{r}^{2}=0,84$ para la variación de masa de sólidos $\left(\Delta \mathrm{M}_{\mathrm{ss}}\right)$ para la temperatura de $30^{\circ} \mathrm{C}$. Entonces, de acuerdo a los resultados de otros estadísticos, con valores de $\mathrm{SSE} \leq 0,0018$; RMSE $\leq 0,0301$ y $\chi^{2} \leq 0,0010$ se puede decir, que éste modelo mostró una buena calidad de ajuste sobre la variación de masa de agua $\left(\Delta \mathrm{M}_{\mathrm{w}}\right)$ y de sólidos solubles $\left(\Delta \mathrm{M}_{\mathrm{ss}}\right)$. Con respecto al modelo difusional, se puede decir que es una buena herramienta de cálculo para predecir la variación de masa de agua $\left(\Delta \mathrm{M}_{\mathrm{w}}\right)$ y de sólidos solubles $\left(\Delta \mathrm{M}_{\mathrm{ss}}\right)$, pero de acuerdo a los resultados de los estadísticos sobre éste modelo, se obtienen valores más altos de $\operatorname{SSE} \geq 0,3463$; RMSE $\geq 0,5885$ y $\chi^{2} \geq 0,4156$, y mas bajos de $\mathrm{r}^{2} \leq 0,84$, que con el modelo de Biswal-Bozorgmehr modificado, por lo cual hace que éste último sea más confiable a la hora de predecir la cinética de transferencia de materia en el fenómeno de la deshidratación osmótica de la papaya chilena.

\subsection{Análisis de regresión lineal múltiple}

Se realizó una regresión lineal múltiple, con el fin de describir la relación existente de las constantes cinéticas $\mathrm{K}_{\mathrm{w}} \mathrm{y} \mathrm{K}_{\mathrm{ss}}$ con las variables del proceso: temperatura y concentración, consideradas factores independientes, de donde se obtuvieron las ecuaciones 15 y 16.

$\mathrm{K}_{\mathrm{w}}=0,00927518-0,00019019 * \mathrm{~T}-0,000459589 * \mathrm{C}$

$\mathrm{K}_{\mathrm{ss}}=0,00586581-0,000200329 * \mathrm{~T}-0,000259812 * \mathrm{C}$

Del mismo modo, se realizó un modelo de regresión lineal múltiple para ver la influencia de las variables de proceso (temperatura y concentración) sobre las difusividades efectivas de agua y de sólidos, de donde se obtuvieron las ecuaciones $17 \mathrm{y}$ 18. Según se observa, tanto para las difusividades efectivas de agua y de sólidos solubles como para las constantes cinéticas $\mathrm{K}_{\mathrm{w}} \mathrm{y} \mathrm{K}_{\mathrm{ss}}$ en todas estas ecuaciones la variable concentración (C) es de mayor relevancia con respecto a la temperatura (T) de la solución osmótica.

$\mathrm{D}_{\mathrm{we}}=-3,6998 \times 10^{-9}-3,94168 \times 10^{-12} * \mathrm{~T}+1,20678 \times 10^{-10} * \mathrm{C}$ 
$\mathrm{D}_{\text {sse }}=-2,0153 \times 10^{-9}+5,5585 \times 10^{-12} * \mathrm{~T}+5,8663 \times 10^{-11} * \mathrm{C}$

\section{Conclusiones}

Con respecto a los resultados obtenidos, se puede decir que el diseño experimental codificado $(\mathrm{n}=3 ; \mathrm{k}=2)$ representado por los 9 experimentos mostraron un comportamiento lineal, con diferentes velocidades de transferencia de materia tanto de agua como de sólidos solubles.

Los parámetros cinéticos $\mathrm{K}_{\mathrm{w}}$ y $\mathrm{K}_{\mathrm{ss}}$ presentaron cierta dependencia con la temperatura de trabajo, lo cual permitió calcular la energía de activación en función de la concentración utilizada. Los valores de $D_{\text {we }}$ y $D_{\text {sse }}$ obtenidos fueron entre 9,53-40,43 $\mathrm{x}$ $10^{-10} \mathrm{~m}^{2} \cdot \mathrm{s}^{-1}$ y $5,54-19,32 \times 10^{-10} \mathrm{~m}^{2} \cdot \mathrm{s}^{-1}$, respectivamente. Sin embargo, estos parámetros no presentaron dependencia clara con la temperatura de la solución osmótica, pero sí con la concentración.

De acuerdo a los análisis estadísticos, tanto el modelo de Biswal-Bozorgmehr modificado como el Difusional resultaron ser útiles para describir la cinética de transferencia de materia durante la deshidratación osmótica, no obstante el modelo Biswal-Bozorgmehr obtuvo la mejor calidad de ajuste sobre la variación de masa de agua y sólidos solubles para cada uno de los experimentos realizados.

Por último, se ha demostrado que con una concentración del $60 \%$ y una temperatura de $30{ }^{\circ} \mathrm{C}$ de la solución osmótica, se puede acelerar la salida de humedad y la ganancia de sólidos solubles ocurridas durante la deshidratación osmótica de la papaya chilena (Vasconcellea pubescens).

\section{Nomenclatura}

$\Delta \mathrm{M} \quad$ Variación de masa total

$\Delta \mathrm{M}_{\mathrm{w}} \quad$ Pérdida de agua

$\Delta \mathrm{M}_{\mathrm{ss}} \quad$ Ganancia de sólidos solubles

$\mathrm{M}_{\mathrm{t}} \mathrm{y} \mathrm{M}_{0} \quad$ Peso de la muestra a tiempo t y 0 respectivamente (g)

$\mathrm{X}_{\mathrm{wt}} \mathrm{y} \mathrm{X}_{\mathrm{w} 0}$ Fracción de masa de agua a tiempo t y o respectivamente (g agua.g ${ }^{-1} \mathrm{~m} . \mathrm{s}$ )

$\mathrm{X}_{\mathrm{sst}} \mathrm{y}_{\mathrm{ss} 0}$ Fracción de masa de $\mathrm{s}$ ólidos solubles a tiempo t y 0 respectivamente (g sólidos.g ${ }^{-1} \mathrm{~m} . \mathrm{s}$ )

$\mathrm{X}_{\mathrm{we}} \quad$ Fracción de masa de agua en equilibrio ( $\mathrm{g}$ agua.g $^{-1}$ m.s)

$\mathrm{K}_{\mathrm{w}} \mathrm{y} \mathrm{K}_{\mathrm{ss}} \quad$ Constantes cinéticas fenomenológicas

$\mathrm{Y}$ Fuerza impulsora (adimensional)

$\mathrm{Y}_{\mathrm{e}} \quad$ Fuerza impulsora experimental (adimensional)

$\mathrm{T} \quad$ Temperatura $\left({ }^{\circ} \mathrm{C}\right)$

C Concentración de sacarosa (\%)

$\mathrm{D}_{\mathrm{e}} \quad$ Difusividad efectiva $\left(\mathrm{m}^{2} \cdot \mathrm{s}^{-1}\right)$

t Tiempo (s)

L Semi espesor (m)

$\mathrm{D}_{\text {we }} \quad$ Difusividad efectiva de agua $\left(\mathrm{m}^{2} \cdot \mathrm{s}^{-1}\right)$

$\mathrm{D}_{\mathrm{sse}} \quad$ Difusividad efectiva de sólidos solubles $\left(\mathrm{m}^{2} \cdot \mathrm{s}^{-1}\right)$

$\mathrm{D}_{0} \quad$ Constante difusional $\left(\mathrm{m}^{2} \cdot \mathrm{s}^{-1}\right)$

$\mathrm{E}_{\mathrm{a}} \quad$ Energía de activación $\left(\mathrm{kJ} \cdot \mathrm{mol}^{-1}\right)$

$\mathrm{R} \quad$ Constante de los gases ideales $\left(\mathrm{kJ} \cdot \mathrm{mol}^{-1} \mathrm{~K}\right)$

$\mathrm{Y}_{\mathrm{c}} \quad$ Fuerza impulsora calculada (adimensional)
Z Número de constantes

N Número de datos

\section{Agradecimientos}

Los autores agradecen a la Dirección de Investigación de la Universidad de La Serena, Chile, por el financiamiento otorgado al Proyecto DIULS No 220-2-16.

\section{Referencias}

1. ADE-OMOWAYE BIO. et al. Osmotic Dehydration Behavior of Red Paprika (Capsicum Annuum L.). Journal of Food Science, v. 67, n. 5, p. 1790-1796, 2002.

2. ANDRADE, S. et al. Desidratação osmótica do jenipapo (Genipa americana L.). Ciência e Tecnologia de Alimentos, v. 23, n. 2, p. 276-281, 2003.

3. A.O.A.C. "Official Method of Analysis", Association of Official Analytical Chemists ( ${ }^{\circ}$ 934.06). Arlington, USA, 1996.

4. BARAT, J. M.; CHIRALT, A.; FITO, P. Effect of Osmotic Solution Concentration, Temperature and Vacuum Impregnation Pretreatment on Osmotic Dehydration Kinetics of Apple Slices. Food Science Technology, v. 7, n. 5, p. 451-456, 2001.

5. BARAT, J.; GRAU, A.; FITO, P. Deshidratación Osmótica de Alimentos, Universidad Politécnica de Valencia, España, p. 12-35, 1998.

6. CHAVARRO-CASTRILLÓN, L.; OCHOA-MARTÍNEZ, C.; AYAÑAOPONTE, A. Efecto de la madurez, geometría y presión sobre la cinética de transferencia de masa en la deshidratación osmótica de papaya (Carica papaya L., var. Maradol). Ciência e Tecnologia de Alimentos, v. 26, n. 3, p. 596-603, 2006.

7. CORZO, O.; CENTENO, A. E. Superficies de respuesta de la transferencia de masa durante la deshidratación osmótica del melón (Cucumis melo, variedad Edisto). Revista de la Facultad de Farmacia, v. 45, n. 1, p. 54-60, 2003.

8. EL-AOUAR, A.; MURR, F. Estudo e modelagem da cinética de desidratação osmótica do mamão Formosa (Carica papaya L.). Ciência e Tecnologia de Alimentos, v. 23, n. 1, p. 69-75, 2003.

9. GASPARETO, O.; OliVEIRA, E.; MAGAlHAES. Influencia del tratamiento osmótico en el secado de la banana "Nanica" (Musa cavendishii, L.) en secador de lecho fijo. Información Tecnológica, v. 15, n. 6, p. 9-15, 2004.

10. GIRALDO, G. et al. Influence of sucrose solution concentration on kinetics and yield duranting osmotic dehydration of mango. Journal of Food Engineering, v. 58, n. 1, p. 33-43, 2003.

11. LERICI, C. L. et al. Osmotic dehydration of fruit: Influence of osmotic agents on drying behavior and product quality. Journal of Food Science, v. 50, n. 1, p. 1217-1219,1985.

12. MENDOZA, R.; SCHMALKO, M. Diffusion coefficients of water and sucrose osmotic dehydration of papaya. International Journal of Food Properties, v. 5, n. 3, p. 537-546, 2003.

13. OCHOA, C. I.; AYALA, A. Modelos matemáticos de transferencia de masa en deshidratación osmótica. Ciencia y Tecnología Alimentaria, v. 4, n. 5, p. 330-342, 2005.

14. RASTOGI, N. K.; RAGHAVARAO, K. S. M. S. Mass transfer during osmotic dehydration of pineapple: considering Fickian diffusion in cubical configuration. Food Science and Technology, v. 37, n. 1, p. 43-47, 2004. 
15. SABLANI, S. S.; SHAFIUR, M.; Al-SADEIRI, D. S. Equilibrium distribution data for osmotic drying of apple cubes in sugar-water solution. Journal of Food Engineering, v. 52, n. 2, p.193-199, 2002.

16. SACChETTI, G.; GIANOTTI, A.; DALlA ROSA, M. Sucrose salt combined effect on mass transfer kinetics and product acceptability. Study on apple osmotic treatments. Journal of Food Engineering, v. 49, n. 2-3, p.163-173, 2001.

17. SIMAL, S. et al. Dehydration of aloe vera: simulation of drying curves and evaluation of funcional properties. Journal of Food Engineering, v. 43, n. 2, p. 109-114, 2000.
18. VEGA, A.; LEMUS, R. Modelado de la cinética de secado de la papaya chilena (Vasconcellea pubescens). Información Tecnológica, v. 17, n. 3, 2006.

19. ZAPATA, J.; CARVAJAL, L.; OSPINA, N. Efectos de la concentración de solutos y la relación jarabe/fruta sobre la cinética de deshidratación osmótica de papaya en láminas. Intercencia, v. 27, n. 5, p. 1-14, 2002. 